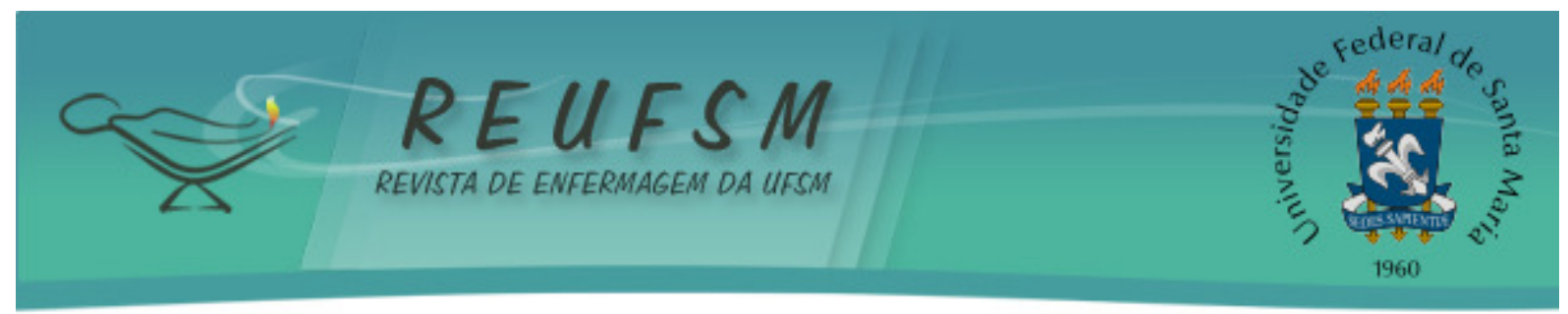

ARTIGO ORIGINAL

\title{
DIARREIA EM CRIANÇAS MENORES DE CINCO ANOS EM UMA UNIDADE DE SAÚDE DA FAMÍLIA
}

\section{DIARRHEA IN CHILDREN YOUNGER THAN 5 YEARS OLD IN A FAMILY HEALTH UNIT \\ DIARREA EN NIÑOS MENORES DE 5 AÑOS EN UNA UNIDAD DE SALUD DE LA FAMILIA}

\author{
Juliana Rogéria Pereira Rodrigues ${ }^{1}$ \\ Laíza Strinta ${ }^{2}$ \\ Grasiela Cristina Silva Botelho Silvestre ${ }^{3}$ \\ Juarez Coimbra Ormonde Junior ${ }^{4}$ \\ Lincoln Christian Barros ${ }^{5}$
}

Doi: $10.5902 / 2179769213490$

RESUMO: Objetivo: caracterizar, por meio da ficha A (cadastro das famílias), prontuários e notificações do Sistema de Vigilância Epidemiológica, os aspectos sociodemográficos e ambientais em crianças menores de cinco anos com diarreia, em uma Estratégia de Saúde da Família. Método: trata-se de um estudo quantitativo com análise documental. A população do estudo constituiu-se de 13 crianças cadastradas em uma estratégia de saúde da família. Resultados: a faixa etária mais acometida por diarreia é a de 1 ano a 1 ano e 11 meses. Houve relativa discrepância entre os dados dos prontuários e os dados do Sistema de Vigilância Epidemiológica em relação ao plano de tratamento oferecido, bem como se constatou a inexistência de prontuários para alguns casos notificados no sistema, - que constitui uma problemática grave que prejudica diretamente a qualidade da assistência prestada. Conclusão: o estudo possibilitou reconhecer o perfil das crianças e identificar problemáticas relacionadas ao registro das informações.

Descritores: Enfermagem; Diarréia infantil; Saúde da criança.

ABSTRACT: Objective: To characterize, by means of form A (registration form of families), medical records and reports of the Epidemiological Surveillance System, sociodemographic and environmental aspects in children under five with diarrhea in the Family Health Strategy. Method: This is a quantitative study with document analysis. The population of the study consisted of 13 children enrolled in a family health strategy. Results: The age group most affected by diarrhea is the one comprising 1 year old to 1 year and 11 months old. There was relative discrepancy between the data from the medical records and the data from the Epidemiological Surveillance System in relation to the treatment plan offered. In addition, there was absence of medical records for some cases reported in the system, which is a severe difficulty that directly affects the quality the profile of the children and the identification of issues related to the registration of information.

Descriptors: Nursing; Diarrhea; Child health.

1 Enfermeira. Especialista em Didática do Ensino Superior. Universidade do Estado de Mato Grosso/UNEMAT. Diamantino (MT), Brasil. E-mail: enf.jurodrigues@gmail.com

2 Enfermeira. Especialista em Obstetrícia e UTI Neonatal e Didática do Ensino Superior. Universidade do Estado de Mato Grosso/UNEMAT. Diamantino (MT), Brasil. E-mail: enf.laizastrinta@hotmail.com

3 Enfermeira. Especialista em Obstetrícia e UTI Neonatal. Universidade do Estado de Mato Grosso/UNEMAT. Diamantino (MT), Brasil. E-mail: grasielabotelho@hotmail.com

4 Enfermeiro. Especialista em Saúde Pública. Universidade do Estado de Mato Grosso/UNEMAT. Tangará da Serra (MT), Brasil. E-mail: coimbra.juarez@gmail.com

5 Enfermeiro. Graduação em Enfermagem. Universidade do Estado de Mato Grosso/UNEMAT. Diamantino (MT), Brasil. E-mail: lincoln-barros@hotmail.com 


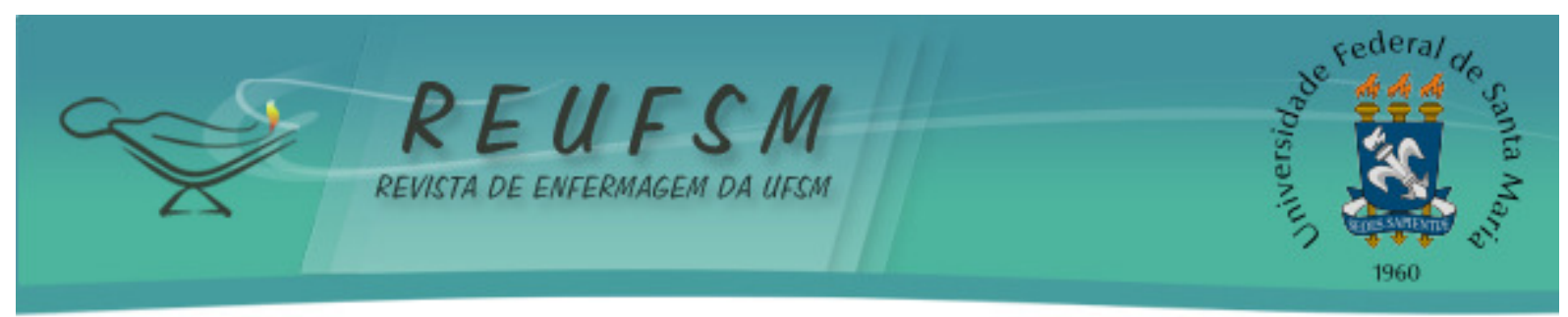

RESUMEN: Objetivo: Caracterizar, mediante los formularios de inscripción de los hogares, los registros e informes del Sistema de Vigilancia Epidemiológica, aspectos sociodemográficos y ambientales en niños menores de cinco años con diarrea, en una Estrategia de Salud Familiar. Método: Estudio cuantitativo de análisis documental. La población estudiada consistió en 13 niños inscritos en una estrategia de salud familiar. Resultados: El grupo de edad más afectado por diarrea es el de 1 año a 1 año y 11 meses. Hubo discrepancia relativa entre los datos de los archivos y los datos del Sistema de Vigilancia Epidemiológica en relación al plan de tratamiento ofrecido, así como la ausencia de registros para algunos casos notificados en el sistema, lo que constituye una problemática grave que afecta directamente a la calidad de la atención prestada. Conclusión: El estudio nos permitió reconocer el perfil de los niños e identificar problemáticas relacionadas con el registro de información.

Descriptores: Enfermería; Diarrea; Salud del niño.

\section{INTRODUÇÃO}

As doenças diarreicas são uma das principais causas de morbidade e mortalidade infantil, situação que acarreta um grande prejuízo no crescimento e desenvolvimento, pois são responsáveis pelo desencadeamento da desnutrição e desidratação, podendo até levar a criança a óbito. De acordo com a Organização Mundial Saúde (OMS), medidas adequadas de saneamento podem reduzir a morbidade pela doença em até $32 \%$, essas condições interferem positivamente na qualidade de vida das populações, principalmente em países em desenvolvimento, onde os investimentos em infraestrutura sanitária encontram-se atrasados em relação a países desenvolvidos. ${ }^{1-2}$

Segundo dados oficiais do Brasil, no período de 1995 a 2005, ocorreram 39.421 mortes por diarreia e 1.505.800 internações associadas a esta doença em crianças menores de cinco anos. A Pesquisa Nacional de Saúde e Nutrição (PNSN) revelou a frequência global de diarreia nas crianças menores de cinco anos de idade de $10,5 \%$, variando de $10 \%$ no meio urbano a $11,6 \%$ no meio rural. $^{3} 0$ elevado índice de óbitos pode estar relacionado diretamente aos fatores sociais ambientais, econômicos e culturais, uma vez que certos grupos populacionais, de menor poder aquisitivo, estariam mais suscetíveis à doença. ${ }^{4}$

Em muitas localidades brasileiras, populações excluídas de infraestrutura urbana, como serviços de saneamento e equipamentos públicos, são atendidas pelo Programa de Saúde da Família (PSF), criado em 1994, como meio de promoção da saúde no país. Nesse programa, as ações voltadas para o atendimento a estas crianças devem ser notificadas ao Sistema de Vigilância Epidemiológica (SIVEP), que tem como objetivo principal gerar um panorama para uma melhor avaliação e tomada de decisão. ${ }^{5}$

0 registro em prontuário fornece subsídios para que sejam realizados planejamentos de acordo com as necessidades da comunidade, visando uma melhor qualidade de vida em sua área de cobertura, tornando-se assim uma ferramenta padrão de coleta de informações da população cadastrada na unidade, algo que deve ser pensado como um requisito básico e essencial para a organização das ações em saúde. Portanto, a qualidade dos registros é fator relevante para a prestação da assistência de enfermagem. ${ }^{6}$

Busca-se somar conhecimentos para a comunidade científica e principalmente aos profissionais de saúde que lidam com este problema no cotidiano. As informações trazidas poderão subsidiar o planejamento e as ações preventivas para a equipe de saúde, com destaque para os Agentes Comunitários de Saúde (ACS), mediadores da equipe de saúde e comunidade, como também aos enfermeiros enquanto parte fundamental do planejamento e organização da unidade de saúde. 


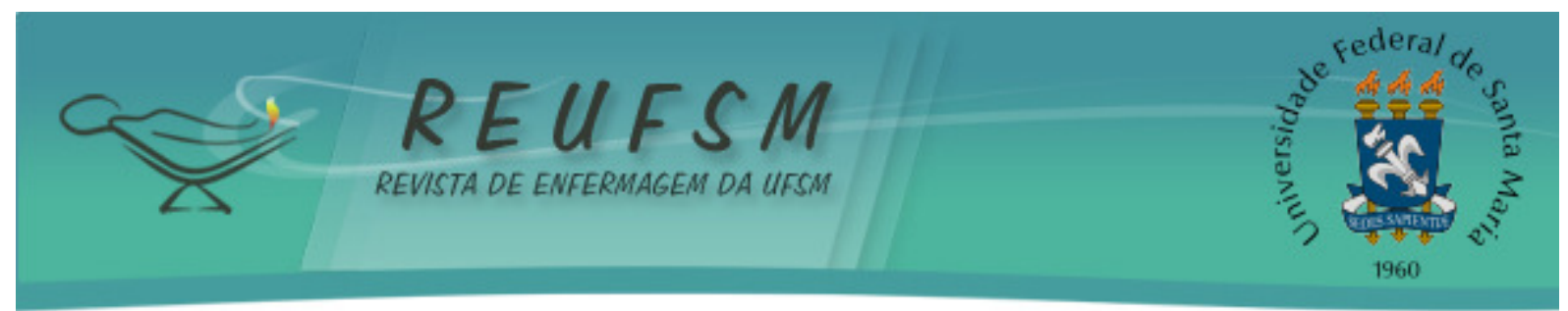

Para tanto, este estudo foi conduzido pela seguinte questão norteadora: com base em documentos específicos disponíveis em uma Estratégia de Saúde da Família, é possível caracterizar os fatores socioeconômicos e ambientais de crianças abaixo de cinco anos acometidas por diarreia? Este estudo teve como objetivo: caracterizar, por meio da ficha A (cadastro das famílias), prontuários e notificações do Sistema de Vigilância Epidemiológica (SIVEP), os aspectos sociodemográficos e ambientais em crianças menores de cinco anos com diarreia, em uma Estratégia de Saúde da Família.

\section{MÉTODO}

Trata-se de um estudo descritivo com abordagem quantitativa, na modalidade de análise documental. Os sujeitos foram 13 crianças menores de cinco anos de idade acometidos por diarreia no ano de 2010, atendidos pela equipe da Estratégia de Saúde da Família (ESF) do bairro Buriti, localizado na periferia do município de Diamantino-Mato Grosso. A assistência à saúde nesse município é constituída por um hospital de pequeno porte e seis Estratégias de Saúde da Família.

Como fontes de informação foram utilizadas a ficha A (formulário de cadastro das famílias), os prontuários de cada sujeito e as informações do Sistema de Vigilância Epidemiológica (SIVEP) do referido município. Foram extraídas as seguintes variáveis na ficha A: sexo, idade, cobertura por ACS, abastecimento de água, destino de lixo, destino das fezes e urina, alfabetização do pai e da mãe, frequência à escola e número de residentes no domicílio. Nos prontuários e no SIVEP foram extraídas as informações referentes à quantidade de casos notificados, bem como o tipo de tratamento oferecido aos pacientes.

Os critérios de inclusão foram: crianças menores de cinco anos, diagnóstico confirmado de diarreia; casos notificados no Sistema de Vigilância Epidemiológica (SIVEP); ficha A devidamente preenchida.

Esse estudo foi realizado após a aprovação do projeto pelo Comitê de Ética em Pesquisa da Universidade de Cuiabá - UNIC, conforme a resolução 196/96 do Conselho Nacional de Saúde. A pesquisa foi norteada de acordo com padrões éticos exigidos com pesquisas envolvendo seres humanos, sob protocolo de número 2011-130, aprovada no dia 26 de agosto de 2011. A coleta de dados foi realizada pelos pesquisadores no período de 01 de setembro de 2011 a 05 de outubro de 2011.

Os dados foram analisados à luz da estatística descritiva e dispostos no software Microsoft Excel 2007, em tabelas e gráficos, no qual permitiu analisar o resultado de frequência absoluta (números de dados) e frequência relativa (porcentagem).

\section{RESULTADOS E DISCUSSÃO}

Neste estudo verificou-se que a maioria da população pesquisada $(n=13)$ é composta por crianças do sexo masculino. Em relação a faixa etária, houve mais comprometimento das crianças de um ano a um ano e onze meses, correspondendo a um total de cinco casos. Para melhor entendimento, apresenta-se na tabela 1 o perfil das crianças acometidas por diarreia. 


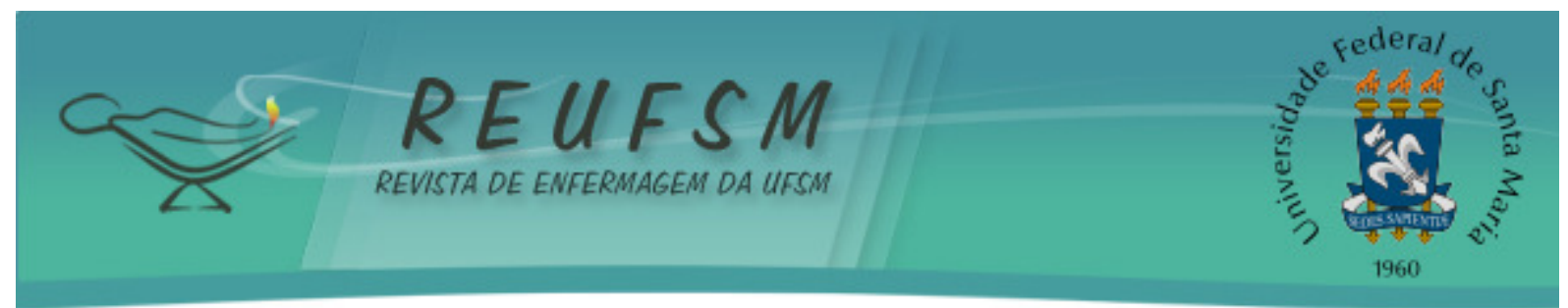

Tabela 1 - Perfil das crianças acometidas por diarreia na Estratégia de Saúde da Família do bairro Buriti. MT, Brasil, 2010.

\begin{tabular}{lc}
\hline & N \\
\hline Sexo & 3 \\
Feminino & 10 \\
Masculino & \\
Faixa etária & 2 \\
0 a 11 meses & 5 \\
1 ano a 1 ano e 11 meses & 1 \\
2 anos a 2 anos e 11 meses & 2 \\
3 anos a 3 anos e 11 meses & 3 \\
4 anos a 4 anos e 11 meses & \\
Cobertura por ACS & 13 \\
Sim & 0 \\
Não & 12 \\
Abastecimento de água & 0 \\
Rede Pública & 1 \\
Poço ou Nascente & \\
Não relata & 12 \\
Destino do lixo & 0 \\
Coletado & 0 \\
Enterrado & 1 \\
Céu aberto & \\
Queimado & 0 \\
Destino das fezes e urina & 12 \\
Sistema de esgoto & 1 \\
Fossa & \\
Céu aberto & 9 \\
Alfabetização do Pai & 0 \\
Alfabetizado & 4 \\
Não Alfabetizado & \\
Não relata & \\
Alfabetização da Mãe & \\
Alfabetizada & \\
Não Alfabetizado & \\
\hline
\end{tabular}

As diferenças de sexo e idade são fundamentais para pensar nas necessidades das crianças, pois a criança que não fala precisa de mais cuidados, pelo fato de nem sempre serem compreendidas, adicionalmente, os autores consideram que as crianças nos primeiros cinco anos são mais vulneráveis a doenças. ${ }^{6} 0$ Ministério da Saúde considera importante identificar a idade da criança para que seja oferecido um tratamento adequado de acordo com a faixa etária. ${ }^{7}$

A respeito da influência dos fatores sociais e ambientais no aparecimento da diarreia, a Tabela 1 dispõe que todas as crianças possuem a área de sua residência coberta por ACS, para 12 sujeitos a água é fornecida pela rede pública. Quanto ao destino do lixo, registra-se que a maior parte é coletada pela prefeitura, e as fezes e urinas são descartadas nas fossas, tornando-se o meio mais utilizado pela população. A disponibilidade de água em quantidade suficiente nos domicílios é a medida mais eficaz no controle da diarreia infecciosa. É importante a orientação a respeito do destino do lixo, das fezes e o uso das fossas domiciliares adequadas. ${ }^{8}$

Crianças que vivem em ambientes menos favoráveis ou em domicílios em condições precárias de saneamento básico são mais suscetíveis ao desenvolvimento da diarreia. Sabe-se que essas condições ambientais desempenham um importante papel no 


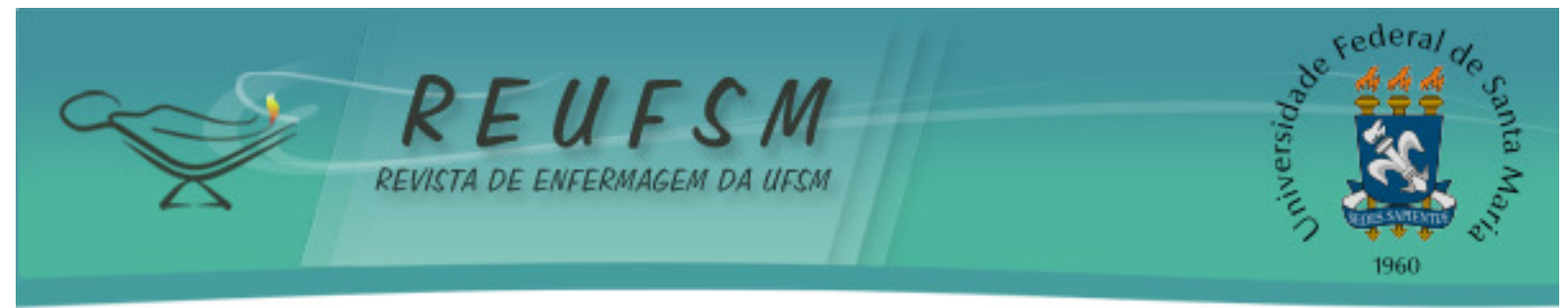

nível de qualidade de vida pela proteção resultante dos serviços sanitários. 0 abastecimento de água e coleta de lixo adequado diminui consideravelmente a morbidade por doenças infecciosas como a diarreia. ${ }^{3}$

$\mathrm{Na}$ Tabela 2 são apresentados os fatores socioambientais em que estão expostas as crianças do estudo.

Tabela 2 - Relação dos fatores sociais e ambientais no surgimento da diarreia. MT, Brasil, 2010.

\begin{tabular}{ll}
\hline & N \\
\hline $\mathbf{N}^{\circ}$ de residentes do domicílio & 5 \\
3 & 3 \\
4 & 3 \\
5 & 1 \\
6 & 1 \\
8 & \\
Frequenta Instituição & 4 \\
Escola & 3 \\
Ambiente institucional - Creche & 5 \\
Não & 1 \\
Não relata & \\
\hline
\end{tabular}

Quanto ao número de residentes, verifica-se que cinco possuem três moradores na mesma residência. Em relação à criança frequentar instituição de ensino, observa-se que cinco não frequentam instituições de ensino e quatro frequentam escolas.

No que se refere à escolaridade das crianças estudadas, de acordo com os dados da Tabela 2, observa-se que grande parte delas frequenta instituições de ensino como escolas e ambientes institucionais.

É importante destacar que as crianças que convivem em ambiente institucional, estão mais sujeitas a agravos de saúde comuns na infância como é o caso da diarreia, sendo esta uma das situações que necessitam de identificação e intervenção precoce para evitar ou minimizar os danos à saúde da criança. Devido ao agrupamento das crianças nos ambientes institucionais, as doenças infectocontagiosas como a diarreia podem proliferar rapidamente, essas doenças ocorrem com mais frequência nos primeiros anos de vida, pois se trata de uma época em que a resistência aos agentes infecciosos pode ser baixa, ao mesmo tempo em que há o aumento do grau de exposição. ${ }^{9}$

O Ministério da Saúde propõe que os profissionais de saúde utilizem o meio institucional para realizar educação em saúde, e com essa estratégia, reduzir o número de casos de diarreia. As ações de saúde, realizadas em espaços coletivos como creches ou escolas, têm um efeito maior do que quando dirigidas individualmente às crianças. Dessa forma, as ações educativas em saúde apresentam resultados muito melhores quando são precedidas de preparo em grupo e realizadas pelos profissionais de saúde de forma integrada com os profissionais da educação. ${ }^{5}$

Acredita-se que esse enfoque ainda seja um desafio para os enfermeiros atuantes na Atenção Primária, mas que deve ser pensada como um processo a ser galgado pelos profissionais de saúde no desenvolvimento de práticas educativas voltadas para a realidade da população. ${ }^{10}$

Das 13 crianças notificadas no SIVEP, apenas seis pacientes possuíam prontuários na ESF e não apresentavam registros nos prontuários. A anotação em prontuário é um instrumento indispensável e é dever do profissional de saúde, pois esse registro confere ao trabalhador a responsabilidade pela assistência prestada ao indivíduo, bem como o respaldo ético e legal. A ficha A é uma ferramenta importante para monitoramento da Estratégia Saúde da Família, serve para juntar todas as informações de saúde das 


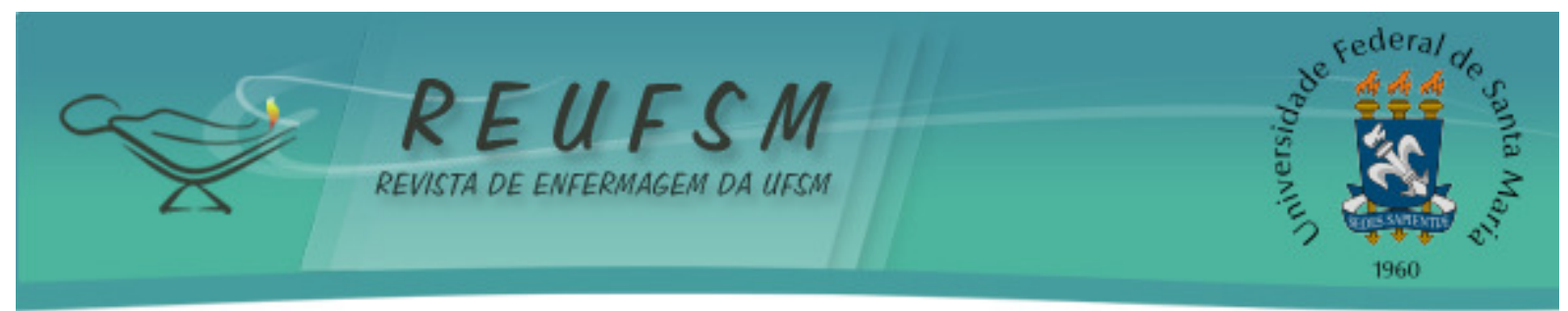

microáreas dos municípios onde atuam os ACS, assim as informações registradas na Ficha A vão para a secretaria de saúde do município, desta, para a secretaria de saúde do estado e, posteriormente, para o Departamento de Atenção Básica do Ministério da Saúde. Esta é uma forma do Governo Federal saber a realidade da saúde das pessoas nos municípios brasileiros e ter mais subsídios para fortalecer a Política Nacional de Atenção Básica. ${ }^{5}$

O ACS ocupa um papel muito importante na equipe, tendo em vista que é este profissional que mantém contato com a população e os profissionais da unidade, trabalhando com a vigilância em saúde, prevenção e promoção da saúde. Em contrapartida, os enfermeiros precisam desenvolver ações básicas de educação em saúde para esses grupos de trabalhadores, com metodologia de como trabalhar em visitas domiciliares, fortalecendo assim a ligação entre o serviço e a comunidade. ${ }^{11}$

Observou-se que em alguns casos a ficha A não existia, em outros estava incompleta. Esta situação observada é relevante, pois vale lembrar que é a partir de tal instrumento que se registram informações importantes sobre as condições de moradia da criança e que são utilizadas para subsidiar ações da unidade, como também para o desenvolvimento do presente trabalho.

Ao realizar a análise das informações dos seis pacientes que possuem prontuário na unidade e compará-las às informações do SIVEP, percebe-se que houve uma relativa discrepância em relação ao plano de tratamento oferecido.

De acordo com os dados dos prontuários, das seis crianças, duas receberam o plano de tratamento $A$, duas receberam o plano de tratamento $B$ e outras duas não relatam o tipo de tratamento oferecido. Segundo os dados obtidos pelo SIVEP, das 13 crianças notificadas com diarreia no bairro Buriti, todas teriam recebido o tipo de tratamento plano A.

$\mathrm{O}$ plano $\mathrm{A}$ é recomendado quando não há sinais e sintomas que indicam a diarreia ou desidratação e constitui-se da administração, na residência do paciente, de alimentos e líquidos. Ò plano B é caracterizado pela utilização de soroterapia oral (SRO), realizada unicamente na ESF quando o paciente apresentar irritação, sede intensa e/ou sinal de prega positiva para desidratação. Quando a criança apresenta sinais que indicam uma desidratação grave, como letargia, olhos fundos e sem brilho, dificuldade para ingestão de líquidos e sinal de prega positivo, o plano C é aplicado. Este último constitui-se de soroterapia endovenosa. ${ }^{12}$

Quando o registro do atendimento é escasso e inadequado, a assistência prestada fica comprometida, uma vez que é com base nesses registros que a prática assistencial poderá de fato ser implementada, pois a escrita é uma das formas mais importantes de comunicação entre a equipe de saúde. ${ }^{13}$

0 prontuário deve conter informações que comprovam o atendimento oferecido ao indivíduo, bem como a escrita deve ser clara e objetiva para qualquer pessoa que leia. Sendo assim, todos da equipe devem prever um determinado tempo para realizar esses registros durante a consulta a cada indivíduo.

O monitoramento e a avaliação das anotações dos profissionais das instituições de saúde são fundamentais, pois visa avaliar a fidedignidade e as limitações de dados em prontuários, sendo uma boa estratégia para melhorar acentuadamente as anotações garantindo qualidade de assistência e melhor gerenciamento de serviço. ${ }^{14}$

Pode-se observar na ESF do bairro Buriti que os registros dos indivíduos são realizados por vários profissionais, como enfermeiros, técnicos de enfermagem e médicos. Também havia informações escassas referentes ao estado clínico da criança, onde os registros existentes faziam maiores referências ao tratamento medicamentoso e aos exames solicitados, não dando devida importância a anotação do histórico e exame físico, podendo assim perceber o tradicional modelo biomédico no atendimento ao sujeito. 


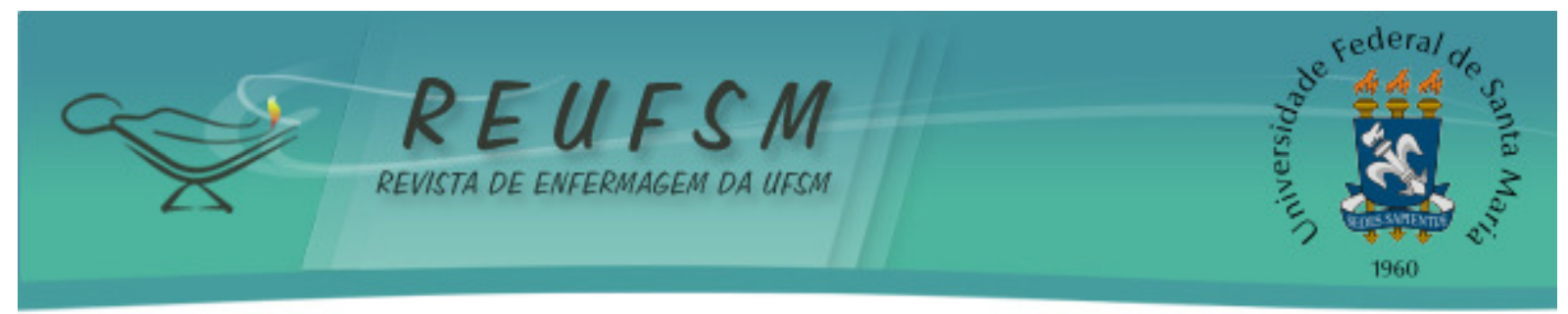

\section{CONSIDERAÇÕES FINAIS}

O estudo permitiu descrever os aspectos sociodemográficos e ambientais dos pacientes menores de cinco anos acometidos por diarreia em uma ESF do bairro buriti. Cabe destacar que, neste estudo, as deficiências no preenchimento das documentações constitui-se de um fator que prejudicou a coleta de maiores informações. Os resultados representam, portanto, a realidade local da unidade de saúde pesquisada.

0 estudo demonstrou que a inexistência de prontuários para alguns casos notificados no SIVEP constitui uma problemática grave que prejudica diretamente a qualidade da assistência prestada. Quando foram comparadas às informações contidas nos prontuários e no SIVEP, particularmente em relação ao plano de tratamento oferecido, houve falta de informações para dos prontuários.

Percebe-se que é necessária a prioridade quanto à qualidade das informações em saúde, no sentido de zelar para a constante notificação da diarreia e do cuidado com estes documentos na própria unidade, estabelecendo assim um bom trânsito destas informações para o nível municipal, para que seja repassado fidedignamente para o nível estadual, assim, perdas de dados serão reduzidas, elevando a qualidade e a credibilidade dos estudos relacionados a estas informações.

Os aspectos sociodemográficos e ambientais descritos nesta pesquisa podem contribuir para um possível planejamento estratégico de ações que visem a redução dos casos de diarreia nessa população. Diante do exposto, espera-se que o presente estudo contribua para que profissionais de saúde e pesquisadores possam refletir a respeito da prevalência dos casos de diarreia em crianças menores de cinco anos, da importância da notificação correta ao SIVEP e do registro correto nos prontuários dos pacientes.

\section{REFERÊNCIAS}

1. Carneiro IA, Sabátes AL. Diarreia na infância. In: Almeida FA, Sabátes AL, organizadores. Enfermagem pediátrica: a criança, o adolescente e sua família no hospital. $1^{\circ}$ ed. São Paulo: Manole; 2008.

2. Oliveira TCR, Latorre MRDO. Tendências da internação e da mortalidade infantil por diarreia: Brasil, 1995 a 2005. Rev Saúde Pública [Internet]. 2010 mar [acesso em 2011 mar 20];44(1):102-11.

Disponível

em:

http: / / www.scielo.br/scielo.php?script=sci_arttext\&pid=S0034-89102010000100011.

3. Benício MHD, César CLG, Gouveia NC. Perfil de morbidade e padrão de utilização de serviços de saúde das crianças brasileiras menores de cinco anos - 1989. In: Monteiro MFG, Iturre RC, Monteiro CA, organizadores. Perfil estatístico de crianças e mães no Brasil: aspectos de saúde e nutrição de crianças no Brasil - 1989. Rio de Janeiro: Fundação IBGE; 1992. p. 79-96.

4. Brasil. Instituto Brasileiro e Geografia e Estatística (IBGE). Censo demográfico 2010 [Internet]. [acesso em 2011 maio 10]. Disponível em: http://www.ibge.gov.br/home/estatistica/populacao/censo2010.

5. Bittar TO, Meneghim MC, Mialhe FL, Pereira AC, Fornazari DH. O Sistema de Informação da Atenção Básica como ferramenta da gestão em saúde. RFO UPF [Internet]. 2009 jan/abr [acesso em 2011 fev 10];14(1):77-81. Disponível em: http://www.upf.br/seer/index.php/rfo/article/view/675/434.

6. Bustamante V, Trad LAB. Cuidando da saúde de crianças pequenas no contexto familiar: um estudo etnográfico com famílias de camadas populares. Ciênc Saúde Coletiva 


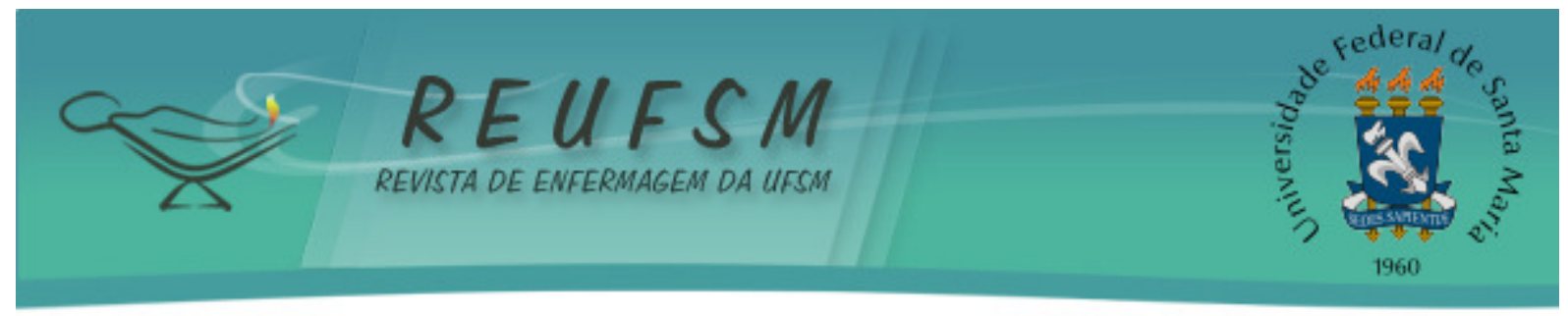

[Internet]. 2007 jun [acesso em 2011 jun 24];12(5):1175-84. Disponível em: http: / /www.scielo.br/scielo.php?script=sci_arttext\&pid=S1413-

$81232007000500014 \& \operatorname{lng}=e n \& n r m=i s s o$.

7. Brasil. Ministério da Saúde. 0 trabalho do agente comunitário de saúde. Brasília (DF): Ministério da Saúde; 2009.

8. Vanderlei LCM, Silva GAP, Braga JU. Fatores de risco para internamento por diarreia aguda em menores de dois anos: estudo de caso-controle. Cad Saúde Pública [Internet]. $2003 \mathrm{abr}$ [acesso em 2011 mar 25];19(2):455-63. Disponível em: http: //www.scielo.br/pdf/csp/v19n2/15411.pdf.

9. Souza MJ, Pinto JP. Agravos a saúde das crianças durante a sua permanência na creche. Rev Soc Bras Enferm Ped [Internet]. 2005 jul [acesso em 2011 maio 8];5(1):27-30. Disponível em: http://www.sobep.org.br/revista/images/stories/pdf-revista/vol5n1/v.5_n.1-art3.pesq-agravos-a-saude-das-criancas.pdf.

10. Dias GAR, Lopes MMB. Educação e saúde no cotidiano de enfermeiras da atenção primária. Rev Enferm UFSM [Internet]. 2013 set [acesso em 2014 jun 6];3(3):449-60. Disponível em: http://cascavel.ufsm.br/revistas/ojs-

2.2.2/index.php/reufsm/article/view/7846/pdf.

11. Vidal SA, Silva EV, Oliveira MG, Siqueira AM, Felisberto E, Samico I, et al. Avaliação da aplicação da estratégia da Atenção Integrada às Doenças Prevalentes da Infância (AIDPI) por agentes comunitários de saúde. Rev Bras Saúde Matern Infant [Internet]. 2013 abr [acesso em 2014 jun 26];3(2):205-13. Disponível em: http://www.scielo.br/pdf/rbsmi/v3n2/a11v03n2.pdf.

12. Brasil. Ministério da Saúde. AIDPI - Atenção Integrada às Doenças Prevalentes na Infância: curso de capacitação: avaliar e classificar a criança de 2 meses a 5 anos de idade: módulo 2. Brasília (DF): Ministério da Saúde; 2003. (Série F. Comunicação e educação em Saúde).

13. Setz VG, D'Innocenzo M. Avaliação da qualidade dos registros de enfermagem no prontuário por meio da auditoria. Acta Paul Enferm [Internet]. 2009 fev [acesso em 2011 nov 15];22(3):313-17. Disponível em: http://www.scielo.br/pdf/ape/v22n3/a12v22n3.pdf.

14. Luz A, Martins AP, Dynewicz AM. Características de anotações de enfermagem encontrados em auditorias. Rev Eletrônica Enferm [Internet]. 2007 maio [acesso em 2011 nov 19];9(2):344-61. Disponível em: http://www.fen.ufg.br/revista/v9/n2/pdf/v9n2a05.pdf.

Data de recebimento: 10/04/2014

Data de aceite: 29/08/2014

Contato com autor responsável: Juliana Rogéria Pereira Rodrigues

Endereço postal: Rua dos Lírios, 110, Bairro Novo Diamantino. CEP: 78.402-000 Diamantino (MT), Brasil.

E-mail: enf.jurodrigues@gmail.com 